\title{
Advancements in the Diagnosis and Treatment of Differentiated Thyroid Cancer
}

\author{
Brendan C. Stack, Jr., MD, Pennsylvania State University College of Medicine Hershey, Pennsylvania
}

[See related article: $53-56$ ]

REPRINT REQUESTS:

Brendan C. Stack, Jr., MD, FACS

Director of Head and Neck

Oncology/Reconstruction

Pennsylvania State University

College of Medicine

500 University Ave, $\mathrm{H} 091$

Hershey, PA 17033-0850

Email: bstack@psu.edu

\section{KEYWORDS:}

lodine radioisotopes; Radioguided surgery; Thyroid neoplasms;

Radiotherapy
The article, "Recombinant TSH-Stimulated, Radioguided Differentiated Thyroid Carcinoma Surgery," highlights two recent advancements in the diagnosis and treatment of well-differentiated thyroid cancer. The first is the use of thyrogen to stimulate the uptake of ${ }^{131} \mathrm{I}$ in thyroid remnants and/or metastases without induction of a clinical hypothyroid state. Secondly, is the use of an intraoperative gamma detector to find and confirm removal of remnant thyroid tissue with radionuclide uptake.

Thyrogen is now widely used to conduct surveillance scanning in thyroid cancer patients. Gamma probe localization is used for sentinel node identification for breast, lung, and head and neck malignancies, as well as melanoma, and minimally invasive parathyroidectomy. The unique aspect of this report is that the two principles have been united, probably for the first time.

Although these results are quite preliminary, they serve to illustrate that an increased ability to biochemically manipulate and image tissues will potentially enable improved staging and treatment for iodine avid thyroid disease, whether in its primary presentation or in the setting of recurrence. Prospective clinical trials will be required to confirm these initial impressions. 